\title{
Mitigating risk of cardiovascular disease in people living with and beyond cancer
}

Faithfull S, Burton C, Clarke S et al (2017) Mitigating risk of cardiovascular disease in people living with and beyond cancer. Cancer Nursing Practice. 16, I, 18-23. Date of submission: 31 August 2016; date of acceptance: 2I November 2016. doi: I0.7748/cnp.2017.el352

\section{Sara Faithfull}

Professor of cancer nursing practice, University of Surrey

\section{Cathy Burton}

Cancer lead for NHS Lambeth

Clinical Commissioning

Group (CCG) and GP adviser,

Macmillan Cancer Support

\section{Sinead Clarke}

Cancer lead for NHS South

Cheshire CCG and GP adviser,

Macmillan Cancer Support

\section{Mike Kirby}

Visiting professor

to the Faculty of Health

and Human Sciences,

University of Hertfordshire

and the Prostate Centre

\section{Alex Lyon}

Consultant cardiologist, Royal Brompton \& Harefield NHS Foundation Trust and senior lecturer, Imperial College London

\section{Gill Levitt}

Honorary paediatric oncologist, Great Ormond Street Hospital for Children NHS Foundation Trust, London

\section{Karen Poole}

Research fellow,

School of Health Sciences,

University of Surrey

\section{Fiona Walter}

Principal researcher in primary care cancer research Department of Public Health and Primary Care, University of Cambridge

\begin{abstract}
Rates of cancer survival have increased in recent decades as a result of earlier diagnosis and improved therapies, but a longer lifespan does not necessarily equate to a healthier life. Chronic illness as a consequence of cancer and its treatment is reported in a significant proportion of people living with and beyond the disease. An increased risk of cardiovascular diseases, such as myocardial infarction, stroke and heart failure, is one of the side effects of some cancer therapies.

Nurses in oncology and primary care can minimise cardiovascular risks by improving people's awareness of symptoms, conducting health assessments and making appropriate referrals. Secondary prevention through lifestyle advice, smoking cessation and obesity reduction is also essential. This should be in combination with more detailed cardiac assessment for those in high-risk groups at all stages of the patient pathway.

Appropriate risk management and early detection of heart problems can prevent long-term illness and reduce multimorbidity for people living with and beyond cancer.
\end{abstract}

\section{Keywords}

cancer, cardiotoxicity, living with and beyond cancer, secondary prevention, side effects, survivorship

CANCER SURVIVAL rates have increased in recent decades with more than 2.5 million people living with or beyond a diagnosis in the UK (Macmillan Cancer Support 2016). More than $50 \%$ of all cancer patients will survive for ten years or more (Coleman et al 2011), rising to more than $80 \%$ of those diagnosed with testicular cancer, malignant melanoma, prostate cancer, breast cancer, Hodgkin lymphoma, and haematological cancers in children and young adults (Cancer Research UK 2016). Therefore, as cases of cancer continue to rise and cancer deaths fall, the number of people living with and beyond the disease will grow (Quaresma et al 2015). Increased survivorship has led to a higher incidence of late effects of treatment especially with multimodal therapies (Elliott et al 2011). Analysis of registry data shows that 1.8 million people living with cancer have at least one other long-term condition such as hypertension, cardiovascular or chronic kidney disease (Maddams et al 2012), which can make them more susceptible to cardiotoxicity. These risks are of particular concern to those who are older and already have existing cardiovascular risk factors (Kalsi et al 2014), or who had received cancer treatment when they were children or young adults (Lipshultz et al 2013).

The extent of cardiac problems in those living with and beyond cancer is difficult to determine. This is because of the way disease and mortality are registered in the UK and the fact that death or referral for heart problems can come many years after a cancer diagnosis. Although severe cardiotoxicity is relatively rare, as reported in clinical trials, analysis from large observational studies shows an increased risk of cardiovascular mortality in many patient groups; for some this is $10 \%$ to $30 \%$ greater than in age-matched controls (Curigliano et al 2010, Bourke et al 2012, Lenihan et al 2013, Suter and Ewer 2013, Kalsi et al 2014, Taylor and Kirby 2015). The reporting of cardiotoxicity varies across studies, partly because those who enter clinical trials are fitter and the short follow-up of many clinical trials compared with cumulative risk of observational studies reflects the wider risks of comorbidity (Cardinale et al 2016) (Table 1). Therefore, the prevalence of cardiotoxicity in a general cancer population is still relatively undefined.

Furthermore, there are a growing number of people who have cardiovascular risk factors before they are diagnosed with cancer. For 
example, $67 \%$ of men and $57 \%$ of women in the UK are overweight (Health and Social Care Information Centre 2015) and these numbers will continue to rise. As a consequence more people are likely to have comorbidities when diagnosed with cancer, and heart health will be a continuing and growing problem for cancer practitioners (Koene et al 2016).

Nurses can help to improve cardiovascular outcomes for cancer patients and people living with and beyond disease. This article identifies cancer treatments that have cardiac consequences as well as assessment and risk management strategies that can be used in clinical practice in oncology and in primary care settings. There is a need to develop interdisciplinary expertise so the best therapeutic opportunities can be provided for people with cancer.

\section{Background}

Some cancer treatments can have cardiovascular consequences by either directly affecting heart physiology or indirectly through increasing cardiovascular risk factors (Box 1). Direct effects of cytotoxic drugs or radiotherapy include acute coronary syndromes, hypertension, arrhythmias, symptomatic left ventricular dysfunction and subsequent heart failure (Lenihan et al 2013, Hamo and Bloom 2015). Indirect effects are caused by drugs that block or influence the metabolic pathways and can speed up atheroma formation or thromboembolism. With the combination of cancer treatments, the effects on the heart can be direct and indirect and have a physical effect resulting in longterm heart health consequences (Box 1).

\section{Direct damage}

Direct damage by chemotherapy drugs is associated most commonly with anthracyclines that cause oxidative distress through damage to the mitochondria and cardiomyocytes (Cardinale et al 2010). These drugs are often used to treat people with leukaemia, lymphoma, breast, uterine, lung, ovarian and childhood cancers. The cardiotoxic effects are dose dependent and irreversible. Exposure to doses greater than $250 \mathrm{mg} / \mathrm{m} 2$ or $300 \mathrm{mg} / \mathrm{m} 2$ can result in dilated cardiomyopathy that may not be detected for many years, or even decades, after treatment (Khawaja et al 2014).

Many newer novel drugs may interfere with intracellular mechanisms that are needed to maintain normal heart cells. For example, trastuzumab, which belongs to the class of monoclonal antibodies that target human epidermal growth factor receptor- 2 and can lead to subclinical cardiac health problems (Lenihan et al 2013, Moslehi 2016) (Table 2). Cancer therapies such as radiotherapy may also cause direct functional vascular changes and inflammation such as pericarditis, but these only occur when radiation therapy is near to the heart (Yeboa and Evans 2016).

\section{Indirect damage}

Indirect damage can occur through the increase in cardiovascular risk factors such as damage to endothelial function, increasing atherosclerosis and increasing the risk of thromboembolism. Anti-hormonal therapies such as those used for breast and prostate cancer can have metabolic effects on testosterone or oestrogens causing an early menopause that can accelerate atheroma formation and result in ischaemic heart disease (Romo et al 2015, Zagar et al 2016). Hormone-modifying therapy can induce dyslipidaemia, high plasma glucose, elevated

BOX 1. Direct and indirect effects of
cancer therapies on the heart
Direct effects
" Valvular heart disease
" Pericardial infusion and constriction
» Conduction system disease
" Loss of myocardium
" Myocardial dysfunction
Indirect effects
" Arterial hypertension
" Impaired endothelial function
" Atherosclerosis
" Arterial thrombosis

\section{Correspondence}

s.faithfull@surrey.ac.uk

\section{Peer review}

This article has been subject to double-blind peer review and checked for plagiarism using automated software

Conflict of interest

The authors are contributors to the Macmillan Cancer Support heart health expert panel

\begin{tabular}{|c|c|c|}
\hline Cancer diagnosis and treatment & Cardiotoxicity & Adjusted hazards ratio \\
\hline $\begin{array}{l}\text { Breast cancer } \\
\text { (Khan et al 2011) }\end{array}$ & $\begin{array}{l}\text { Heart failure } \\
\text { Coronary heart disease }\end{array}$ & $\begin{array}{l}1.95 \\
1.27\end{array}$ \\
\hline $\begin{array}{l}\text { Prostate cancer } \\
\text { (Keating et al 2010) }\end{array}$ & $\begin{array}{l}\text { Myocardial infarction } \\
\text { Coronary heart disease } \\
\text { Sudden cardiac death } \\
\text { Stroke }\end{array}$ & $\begin{array}{l}1.28 \\
1.19 \\
1.35 \\
1.22\end{array}$ \\
\hline $\begin{array}{l}\text { Lymphoma } \\
\text { (Armenian et al 2016) }\end{array}$ & Vascular heart disease & 2.3 \\
\hline $\begin{array}{l}\text { Survivors of cancer when a child } \\
\text { or young adult } \\
\text { (Mulrooney et al 2009) }\end{array}$ & $\begin{array}{l}\text { Congestive heart failure } \\
\text { Myocardial infarction } \\
\text { Pericardial disease } \\
\text { Valvular abnormalities }\end{array}$ & $\begin{array}{l}5.9 \\
5 \\
6.3 \\
4.8\end{array}$ \\
\hline
\end{tabular}


insulin levels, elevated total and low-density lipoprotein cholesterol, elevated triglycerides and decreased liver function. These are many of the components of metabolic syndrome, which increases long-term cardiovascular risk (Redig and Munshi 2010, Bourke et al 2012). Androgen deprivation therapy also decreases lean body mass, increases fat mass and increases arterial stiffness (Edelman et al 2014). These effects can be seen quickly and occur within three months of the initiation of therapy.

Some cardiac effects are reversible and others are cumulative and dose dependent, although this distinction oversimplifies the subclinical changes. By the time people who have had cancer are symptomatic, cardiac damage can be irreversible (Monsuez et al 2010).

Part of the acute oncology team's role is to assess previous cardiovascular health, as well as mitigate potential risks when making treatment choices (Albini et al 2010, Kirby 2016). However, ongoing management and support for people living with and beyond cancer falls between many practitioners. In a recent UK survey of oncology and community nurses, only $16 \%$ of nurses and allied health professionals felt confident to assess cardiovascular problems related to cancer therapy (Faithfull et al 2016). GPs also have a lack of knowledge in this area. Only $21 \%$ of GPs considered a history of cancer when assessing cardiovascular illness, with variability in their awareness of the heart-related consequences of different cancer therapies; $40 \%$ were aware of the effect of radiotherapy, $53 \%$ of hormone therapy and $50 \%$ of chemotherapy (Walter et al 2015). Nurses and GPs in both studies wanted to learn more about how they could improve outcomes for people living with and beyond a cancer diagnosis and treatment.

Addressing this knowledge gap for people with cancer and clinicians has been one of the goals of the Macmillan Cancer Support heart health expert panel, who have published a quick guide for clinicians (Macmillan Cancer Support 2015a) and a self-help guide for people living with and beyond cancer (Macmillan Cancer Support and British Heart Foundation 2014, Macmillan Cancer Support 2015b).

The recovery package offers a framework of care and support for people after treatment for cancer and is part of the cancer strategy to improve outcomes (Independent Cancer Taskforce 2015). This package identifies the requirement for holistic needs assessment and care planning, treatment summary, health and well-being events, and cancer care review. After treatment people living with and beyond cancer should receive a follow-up appointment with their GP to help them with rehabilitation, support and self-management. This cancer review is an opportunity to identify potential side effects, address existing symptoms and promote health and lifestyle advice. GPs can only provide this advice if treatment summaries identify potential heart health issues. One of the barriers to awareness is a belief that unnecessarily worrying people with cancer about long-term effects of therapy may cause distress. Evidence suggests that this is not the case (Cox and Faithfull 2013) and is reflected in studies that report satisfaction with information provision in people living with and beyond cancer. People with Hodgkin lymphoma who were assessed for cardiovascular risks and participated in a recall screening and counselling service found that the additional information improved satisfaction scores (Daniëls et al 2014). The benefits of screening were seen by people with cancer to outweigh the burden of assessment.

In a further study exploring information satisfaction over time, satisfaction with

\section{TABLE 2. Cancer therapies that cause cardiotoxicity}

\begin{tabular}{l} 
Class of cancer drugs or therapy \\
Anthracyclines \\
Alkylating agents \\
Targeted therapies: monoclonal antibodies \\
\hline
\end{tabular}

Small molecule tyrosine kinase inhibitors

Hormonal therapies

\section{Examples}

Doxorubicin, epirubicin, idarubicin, mitoxantrone Cyclophosphamide

Trastuzumab, bevacizumab

Imatinib, sorafenib, sunitinib, mesylate

Anti-oestrogens: tamoxifen, anastrozole and letrozole Anti-androgens: goserelin and buserelin Surgical orchidectomy

Radiotherapy involving the heart

(Adapted from Zamorano et al 2016)
Left breast or mediastinal radiotherapy fields 
information provision was again better at baseline than at follow-up and satisfaction was associated with better emotional and cognitive functioning in those living with and beyond cancer (Husson et al 2014). Improving awareness through information and tailoring information to specific needs helps people with cancer understand their illness better and also brings health benefits (Husson et al 2013).

\section{Assessment}

Assessment and risk stratification are critical to knowing who might need what information and supportive care. One of the challenges is accurately stratifying people according to their cardiac risk; there is no reliable surrogate marker to predict long-term cardiovascular outcomes in people living with and beyond cancer and the longer term risks of novel therapies and multimodal treatments are as yet unknown. Echocardiography is a key investigation when assessing heart health, especially in people with cancer, as it can be used as a baseline assessment of cardiac function and to diagnose any unrecognised cardiac problems (Lenihan et al 2013, Suter and Ewer 2013). Ideally, people at highest risk (Macmillan Cancer Support 2015a) should have a baseline echocardiogram and reassessments six months after the end of treatment if they are not symptomatic, and subsequently every five years as part of planned surveillance. Not all centres have access to echocardiography, however. The alternative electrocardiogram, combined with cardiac biomarkers B-type natriuretic peptide and troponin, can be good indicators of cardiac abnormality and should trigger referral for further tests if abnormal (Kirby 2016).

Nurses can assess people with cancer during and after treatment through observations, including measuring blood pressure, pulse for arrhythmias, body mass index, waist to hip ratio, in addition to using validated cardiac risk calculators such as QRISK 2 or JBS3 in conjunction with the National Institute for Health and Care Excellence (2014) guideline on cardiovascular disease. These online tools give a ten-year risk assessment and, although not specifically designed for people with cancer, they can raise awareness of how healthy lifestyle choices can reduce overall cardiovascular risk. Individualised risk assessments taking into account genetic predispositions, co-morbidities, cholesterollowering and smoking cessation behaviours, and physical activity are essential for the prevention and management of cardiac problems. Enquiring about erectile dysfunction in men before cancer treatment can be a marker of existing endothelial dysfunction and vascular risk and prompt further assessment.

Nurses can ask about potential cardiac symptoms such as fatigue and shortness of breath on exertion, exercise intolerance and functional changes since treatment, such as difficulty climbing stairs, which can be an early indicator of cardiovascular disease.

\section{Prevention and management}

Raising awareness is critical in prevention. A cancer diagnosis can be a 'teachable moment' where people reflect on their lives and are more likely to adopt a healthier lifestyle. Providing information and support for people considering treatment options, especially those at higher risk of cardiovascular disease as defined by Macmillan Cancer Support (2015a), could prevent future occurrence of chronic illness. Such information could include weight loss, hypertension management or smoking cessation. For some people their risk of cardiac disease will outweigh their risk of cancer recurrence.

Prevention of future cardiovascular consequences through optimal treatment and management is a focus of research and development. Despite therapeutic advances and improved survivorship, people living with and beyond cancer will continue to have cardiovascular disease as a consequence of changing therapies and pre-existing co-morbidities. Prevention is also about promoting healthy lifestyle choices to reduce indirect risks. The World Health Organization (Stewart and Wild 2014) estimates that approximately $30 \%$ of cancer deaths could be prevented by modifying or avoiding risk factors such as smoking, obesity and unhealthy diets low in fruit and

Figure I. Identification of heart health risk factors and methods to reduce risk

\begin{tabular}{|c|c|c|c|}
\hline Genetics & Existing co-morbidity & & $\begin{array}{l}\text { Reduce existing risks by } \\
\text { promoting healthy lifestyle }\end{array}$ \\
\hline $\begin{array}{l}\text { Family history of } \\
\text { cardiovascular disease before } \\
\text { the age of } 50 \text { years }\end{array}$ & $\begin{array}{l}\text { Type } 2 \text { diabetes } \\
\text { Rheumatoid arthritis } \\
\text { Existing cardiovascular disease } \\
\text { Communicate what the likely } \\
\text { treatment effects are and } \\
\text { how this may interact with } \\
\text { comorbidities } \\
\text { Review comorbidities when } \\
\text { having therapy for recurrence }\end{array}$ & $\rightarrow$ & $\begin{array}{l}\text { Promote smoking cessation } \\
\text { Advise to keep physically active } \\
\text { Advise a Mediterranean style } \\
\text { diet rich in fruit, vegetables, oily } \\
\text { fish and wholegrain cereals, } \\
\text { low in dairy fats and red meat } \\
\text { Recommend NHS alcohol advice } \\
\text { Maintain a healthy weight }\end{array}$ \\
\hline
\end{tabular}

FIND OUT MORE

» Macmillan Cancer Support and British Heart Foundation (2014) Heart Health and Cancer Treatment. http://be.macmillan. org.uk/Downloads/ LivingWithAndAfter Cancer/MACl4637 Hearthealthand cancertreatment Ellowrespdfnewcover

" Macmillan Cancer Support (2015) Managing Heart Health During and After Cancer Treatment: A Quick Guide for Primary Care Health Professionals.

www.macmillan. org.uk/documents/ aboutus/health_ professionals/ consequence softreatment/ hearthealthguide.pdf

» Macmillan Cancer Support (2015) Tips for Managing Heart Health During and After Cancer Treatment.

www.macmillan. org.uk/documents/ aboutus/health_ professionals/ consequencesof treatment/ healthhealthtips.pdf CancerInformation/ SH20160217.pdf 
vegetables and low levels of physical activity. Addressing these modifiable factors (Figure 1) (Kirby 2016) is critical to reduce the overlap between comorbidities and treatment-related cardiovascular toxicity (Koene et al 2016). There is evidence that these interventions can also reduce cancer recurrence as well as the severity of recurrence (Kenfield et al 2011). Advice for people living with and beyond cancer and who have a cardiovascular risk is: to eat a healthy Mediterranean-style diet, that is one rich in fruit and vegetables, oily fish and wholegrains and low in fat, dairy foods and red meats; maintain a healthy weight; keep physically active; stop smoking; and avoid too much alcohol and binge drinking.

\section{Clinical implications}

Effective communication with primary and secondary care colleagues is necessary to identify previous comorbidities that might affect treatment choices but also to monitor long-term cardiovascular health in people with cancer. Providing information on potential cardiovascular risks and alerting primary care teams to the need for heart health intervention is essential. Referral to secondary care cardiology services should occur with people with cancer who have abnormal cardiac function or cardiovascular symptoms during surveillance (Table 3). Management interventions follow the standard heart failure guidelines but more research is needed on cardioprotection and the development of best care approaches for managing people living with and beyond cancer who have cardiotoxicities. Nurses have an essential role in prevention, early recognition and management of cardiac symptoms.

\section{TABLE 3. Criteria for referral to secondary care cardiology services}

\section{Stage of cancer pathway}

Diagnosis and treatment

Recovery phase

Surveillance
People with abnormal cardiac function who will receive cardiotoxic cancer therapy.

Any new cardiovascular abnormality in symptomatic people with cancer who have established cardiovascular disease.

Women who were treated with cardiotoxic chemotherapy or radiotherapy and are planning a pregnancy.

People living with and beyond cancer treatment who were treated with cardiotoxic therapy and who wish to participate in intensive exercise.

\section{Conclusion}

Nurses play a major role in holistic assessment and symptom management for people living with and beyond cancer, either in the oncology or primary care setting. Recognising the growing cardiac burden for people with cancer during and after treatment is a shared responsibility. Some older people may already have comorbidities before their cancer treatment starts so it is important that they are well informed, prevention and risk reduction is in place, and that if cardiac problems occur that they are diagnosed early. We also need to understand how emerging therapies affect individuals in causing cardiotoxicity so that the clinical team can be vigilant. The end of treatment review is a good opportunity to discuss potential long-term cardiac risks, provide lifestyle advice and information. Comprehensive assessment and early recognition are part of the recovery pathway but patient-reported outcomes alone may not detect rising blood pressure or that fatigue and breathlessness may be a result of heart failure. Working in collaboration with primary care teams is therefore essential.

\section{Implications for practice}

»Direct and indirect cardiac consequences of treatment are an increasingly significant problem for people living with and beyond cancer.

» Guidance developed by Macmillan Cancer Support on managing heart health during and after cancer treatment, as well as top tips, is available to inform practice.

» Healthcare professionals in oncology and general practice settings need to be aware of and actively develop risk management strategies and appropriate interventions to detect and prevent cardiac consequences.

»People with cancer need to be aware of their personal cardiovascular risk and how their lifestyle choices, together with medical surveillance where appropriate for those at highest risk, can mitigate this risk. 


\section{References}

Albini A, Pennesi G, Donatelli F et al (2010) Cardiotoxicity of anticancer drugs: the need for cardio-oncology and cardio-oncological prevention. Journal of the National Cance Institute. 102, 1, 14-25.

Armenian S, Xu L, Ky B et al (2016) Cardiovascular disease among survivors of adult-onset cancer: a community-based retrospective cohort study. Journal of Clinical Oncology. 34, 10, 1122-1130.

Bourke L, Chico T, Albertsen P et al (2012) Cardiovascular risk in androgen suppression: underappreciated, under-researched and unresolved. Heart. 98, 5, 345-348.

Cancer Research UK (2016) Cancer Statistic for the UK. www.cancerresearchuk.org/healthprofessional/cancer-statistics (Last accessed 25 November 2016.

Cardinale D, Colombo A, Lamantia G et al (2010) Anthracycline-induced cardiomyopathy: clinical relevance and response to pharmacologic therapy. Journal of the American College of Cardiology. 55, 3, 213-220

Cardinale D, Biasillo G, Cipolla C (2016) Curing cancer, saving the heart: a challenge that cardioncology should not miss. Current Cardiology Reports. 18, 6, 51

Coleman M, Forman D, Bryant $\mathrm{H}$ et al (2011) Cancer survival in Australia, Canada, Denmark, Norwa Sweden and the UK, 1995-2007: an analysis of population-based cancer registry data. Lancet 377, 9760, 127-138.

Cox A, Faithfull S (2013) 'They're survivors physically but we want them to survive mentally as well': health care professionals' views on providing potential late effect information. Supportive Care in Cancer. 21, 9, 2491-2497.

Curigliano G, Mayer E, Burstein H et al (2010) Cardiac toxicity from systemic cancer therapy: a comprehensive review. Progress in Cardiovascular Diseases. 53, 2, 94-104.

Daniëls L, Krol S, de Graaf M et al (2014) Impact of cardiovascular counseling and screening in Hodgkin lymphoma survivors. International Journal of Radiation Oncology, Biology, Physics. 90, 1, 164-171.

Edelman S, Butler J, Hershatter B et al (2014) The effects of androgen deprivation therapy on cardiac function and heart failure: implication for management of prostate cancer. Clinica Genitourinary Cancer. 12, 6, 399-407

Elliott J, Fallows A, Staetsky L et al (2011) The health and well-being of cancer survivors in the UK: findings from a population-based survey. British Journal of Cancer. 105, Suppl 1, S11-S20.
Faithfull S, Samuel C, Lemanska A et al (2016) Selfreported competence in long term care provision for adult cancer survivors: a cross sectional survey of nursing and allied health care professionals. International Journal of Nursing Studies. 53, 85-94.

Hamo C, Bloom M (2015) Getting to the heart of the matter: an overview of cardiac toxicity related to cancer therapy. Clinical Medicine Insights. Cardiology. 9, Suppl 2, 47-51

Health and Social Care Information Centre (2015) Statistics on Obesity, Physical Activity and Diet: England 2015. http://content.digital.nhs. uk/catalogue/pub16988/obes-phys-acti-dieteng-2015.pdf (Last accessed: 25 November 2016.

Husson 0, Thong M, Mols F et al (2013) Illness perceptions in cancer survivors: what is the role of information provision? Psycho-oncology. 22 3, 490-498

Husson 0, 0erlemans S, Mols F et al (2014) Satisfaction with information provision is associated with baseline but not with follow-up quality of life among lymphoma patients: results from the PROFILES registry. Acta Oncologica. 53, 7, 917-926.

Independent Cancer Taskforce (2015) Achieving World-Class Cancer Outcomes: A Strategy fo England 2015-2020. NHS, England.

Kalsi T, Babic-Illman G, Fields P et al (2014) The impact of low-grade toxicity in older people with cancer undergoing chemotherapy. British Journal of Cancer. 111, 12, 2224-2228.

Keating N, O'Malley A, Freedland S et al (2010) Diabetes and cardiovascular disease during androgen deprivation therapy: observational study of veterans with prostate cancer. Journal of the National Cancer Institute. 102, 1, 39-46.

Kenfield S, Stampfer M, Giovannucci E et al (2011) Physical activity and survival after prostate cance diagnosis in the health professionals follow-up study. Journal of Clinical Oncology. 29, 6,726-732.

Khan N, Mant D, Carpenter L et al (2011) Long-term health outcomes in a British cohort of breast colorectal and prostate cancer survivors: a database study. British Journal of Cancer. 105 Suppl 1, S29-S37.

Khawaja M, Cafferkey C, Rajani R et al (2014) Cardiac complications and manifestations of chemotherapy for cancer. Heart. 100, 14, 1133-1140.

Kirby M (2016) Adding life to years: heart health and cancer. Primary Care Cardiovascular Journal. www.issuesandanswers.org/wp-content/uploads/ MacMillanHeartHealthCancer.pdf (Last accessed: 25 November 2016.
Koene R, Prizment A, Blaes A et al (2016) Shared risk factors in cardiovascular disease and cancer. Circulation. 133, 11, 1104-1114.

Lenihan D, Oliva S, Chow E et al (2013) Cardiac toxicity in cancer survivors. Cancer. 119, Suppl 11, $2131-2142$

Lipshultz S, Adams M, Colan S et al (2013) Long-term cardiovascular toxicity in children, adolescents, and young adults who receive cancer therapy: pathophysiology, course, monitoring management, prevention, and research directions: a scientific statement from the American Heart Association Circulation 128, 17, 1927-1995.

Macmillan Cancer Support and British Heart Foundation (2014) Heart Health and Cancer Treatment. http://be.macmillan.org.uk/Downloads/ CancerInformation/LivingWithAndAfterCancer/ MAC14637Hearthealthandcancertreatment EllowrespdfnewcoverSH20160217.pdf (Last accessed: 25 November 2016.

Macmillan Cancer Support (2015a) Managing Heart Health During and After Cancer Treatment: A Quick Guide for Primary Care Health Professionals. www.macmillan.org.uk/documents/aboutus/ health professionals/consequencesoftreatment/ hearthealthguide.pdf (Last accessed: 25 November 2016.

Macmillan Cancer Support (2015b) Tips for Managing Heart Health During and After Cancer Treatment. www.macmillan.org.uk/ documents/aboutus/health professionals/ consequencesoftreatment/healthhealthtips.pdf (Last accessed: 25 November 2016.)

Macmillan Cancer Support (2016) Cancer: Then and Now. Diagnosis, Treatment and After Care from 1970-2016. www.macmillan.org.uk/documents/ campaigns/cancer-then-now-report-final-online. pdf (Last accessed: 25 November 2016.)

Maddams J, Utley M, Møller H (2012) Projections of cancer prevalence in the United Kingdom, 20102040. British Journal of Cancer. 107, 7, 1195-1202.

Monsuez J, Charniot J, Vignat N et al (2010) Cardiac side-effects of cancer chemotherapy. International Journal of Cardiology. 144, 1, 3-15

Moslehi J (2016) Cardiovascular toxic effects of targeted cancer therapies. The New England Journal of Medicine. 375, 15, 1457-1467

Mulrooney D, Yeazel M, Kawashima T et al (2009) Cardiac outcomes in a cohort of adult survivors of childhood and adolescent cancer: retrospective analysis of the Childhood Cancer Survivor Study cohort. BMJ. 339:b4606
National Institute for Health and Care Excellence 2014) Cardiovascular Disease: Risk Assessment and Reduction, Including Lipid Modification. Clinical Guideline 181. www.nice.org.uk/guidance/ cg181 (Last accessed: 7 December 2016.

Quaresma M, Coleman M, Rachet B (2015) 40-year trends in an index of survival for all cancers combined and survival adjusted for age and sex for each cancer in England and Wales, 1971-201: a population-based study. Lancet. 385, 9974, 1206-1218

Redig A, Munshi H (2010) Care of the cancer urvivor: metabolic syndrome after hormonemodifying therapy. American Journal of Medicine. 123, 1, 87.e1-6.

Romo M, McCrillis A, Brite J et al (2015) Pharmacologic androgen deprivation and cardiovascular disease risk factors: a systematic review. European Journal of Clinical Investigation $45,5,475-484$

Stewart B, Wild C (Eds) (2014) World Cancer Report 2014. International Agency for Research on Cancer, France.

Suter T, Ewer M (2013) Cancer drugs and the heart: importance and management. European Heart Journal. 34, 15, 1102-1111

Taylor C, Kirby A (2015) Cardiac side-effects from breast cancer radiotherapy. Clinical Oncology. 27, $11,621-629$.

Walter F, Usher-Smith J, Yadlapalli S et al (2015) Caring for people living with, and beyond, cancer: an online survey of GPs in England. British Journal of General Practice 65, 640, e761-768.

Yeboa D, Evans S (2016) Contemporary breast radiotherapy and cardiac toxicity. Seminars in Radiation Oncology. 26, 1,71-78

Zagar T, Cardinale D, Marks L (2016) Breast cancer therapy-associated cardiovascular disease. Nature Reviews. Clinical Oncology. 13, 3, 172-184.

Zamorano J, Lancellotti P, Rodriguez Muñoz D et al (2016) 2016 ESC Position Paper on cancer treatments and cardiovascular toxicity developed under the auspices of the ESC Committee for Practice Guidelines. European Heart Journal. doi:10.1093/eurheartj/ehw211 